\title{
Ladislav Bican
}

Completely decomposable abelian groups any pure subgroup of which is completely decomposable

Czechoslovak Mathematical Journal, Vol. 24 (1974), No. 2, 176-191

Persistent URL: http://dml.cz/dmlcz/101232

\section{Terms of use:}

(C) Institute of Mathematics AS CR, 1974

Institute of Mathematics of the Czech Academy of Sciences provides access to digitized documents strictly for personal use. Each copy of any part of this document must contain these Terms of use.

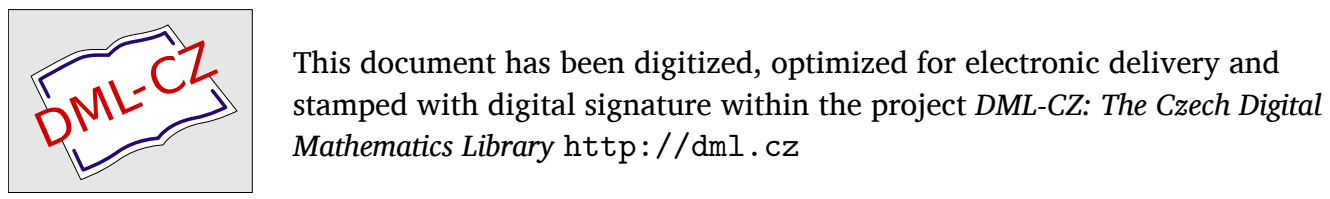




\title{
COMPLETELY DECOMPOSABLE ABELIAN GROUPS ANY PURE SUBGROUP OF WHICH IS COMPLETELY DECOMPOSABLE
}

\author{
Ladislav BicAN, Praha
}

(Received February 13, 1972)

In this paper we shall study the completely decomposable torsion free abelian groups any pure subgroup of which is completely decomposable, again. Concerning the groups the type set of which satisfies the maximum condition we obtain a full description (Theorem 1). For to describe the class of all such groups it suffices to know all such groups the type set of which contains an infinite increasing sequence $\hat{\tau}_{1}<\hat{\tau}_{2}<\ldots$ such that for every $\hat{\tau} \in \hat{\tau}(G)$ there is $\hat{\tau} \leqq \hat{\tau}_{n}$ for some $n$ and the set $\left\{\hat{\tau}, \hat{\tau} \in \hat{\tau}(G), \hat{\tau} \leqq \hat{\tau}_{n}\right\}$ is inversely well ordered for every $n$ (Theorem 2 ).

All the groups considered below will be torsion free abelian groups. In what follows we shall use the following notation: $h_{p}^{G}(g)\left(\tau^{G}(g), \hat{\tau}^{G}(g)\right.$ resp.) denotes the $p$-height (the height, the type resp.) of the element $g$ in the group $G$. If $\tau$ is a height then $\hat{\tau}$ will be the type to which the height $\tau$ belongs. By $T(G)$ we denote the set of the types of all direct summands $J_{\alpha}$ of a completely decomposable group $G=\sum_{\alpha \in A} J_{\alpha}$ (it is wellknown that $T(G)$ is an invariant of $G-$ see Theorem 46.1 in [1]). $\hat{\tau}(G)$ will denote the type set of the group $G$, i.e. the set of the $\hat{\tau}^{G}(g)$ for all $g \in G, g \neq 0$. Other notation and terminology will be essentially that as in [1]. Especially, $r(G)$ is the rank of $G$ and $\hat{\tau}_{1} \| \hat{\tau}_{2}$ denotes the incomparability of the types $\hat{\tau}_{1}, \hat{\tau}_{2}$.

We start our investigations with some preliminary lemmas.

Lemma 1. Let $J_{i}, i=1,2,3$ be three reduced torsion free groups of rank one and of the types $\hat{\tau}_{i}, i=1,2,3$ satisfying $\hat{\tau}_{2} \| \hat{\tau}_{3}, \hat{\tau}_{1} \geqq \hat{\tau}_{2} \vee \hat{\tau}_{3}$. Then the group $G=$ $=J_{1}+J_{2} \dot{+} J_{3}$ contains a pure subgroup which is not completely decomposable.

Proof. Let $p$ be a prime for which $J_{1}$ (and hence $J_{2}, J_{3}$ ) is not $p$-divisible. Let us take the elements $0 \neq u_{i} \in J_{i}, i=1,2,3$, with $h_{p}^{G}\left(u_{i}\right)=0, i=1,2,3$. We are going to show that $S=\left\{u_{1}+p u_{2}, u_{1}+p u_{3}\right\}_{*}^{G}$ is not completely decomposable. Proving indirectly let us suppose $S=I_{1}+I_{2}$. Then necessarily $\hat{\tau}\left(I_{1}\right)=\hat{\tau}_{2}, \hat{\tau}\left(I_{2}\right)=\hat{\tau}_{3}$ and hence $u_{1}+p u_{2} \in I_{1}, \quad u_{1}+p u_{3} \in I_{2}$. Further $p\left(u_{2}-u_{3}\right)=\left(u_{1}+p u_{2}\right)-$ 
- $\left(u_{1}+p u_{3}\right) \in S$, hence $u_{2}-u_{3} \in S, S$ being pure in $G$. Therefore $u_{2}-u_{3}=$ $=h_{1}+h_{2}, h_{1} \in I_{1}, h_{2} \in I_{2}$, from hich $p\left(u_{2}-u_{3}\right)=p h_{1}+p h_{2}=\left(u_{1}+p u_{2}\right)-$ - $\left(u_{1}+p u_{3}\right)$. In view of the form of $S$ we have $p h_{1}=u_{1}+p u_{2}$ which yields a contradiction with $h_{p}^{G}\left(u_{1}\right)=0$.

Lemma 2. Let $J_{i}, i=1,2,3$ be three reduced torsion free group of rank one and of the types $\hat{\tau}_{i}, i=1,2,3$. If any pure subgroup of $G=J_{1}+J_{2}+J_{3}$ is completely decomposable then the set $\left\{\hat{\tau}_{1} \cap \hat{\tau}_{2}, \hat{\tau}_{1} \cap \hat{\tau}_{3}, \hat{\tau}_{2} \cap \hat{\tau}_{3}, \hat{\tau}_{1} \cap \hat{\tau}_{2} \cap \hat{\tau}_{3}\right\}$ is ordered (in the natural order of the types) and contains at most two different elements.

Proof. a) If the types $\hat{\tau}_{1} \cap \hat{\tau}_{2}, \hat{\tau}_{1} \cap \hat{\tau}_{3}, \hat{\tau}_{2} \cap \hat{\tau}_{3}, \hat{\tau}_{1} \cap \hat{\tau}_{2} \cap \hat{\tau}_{3}$ are pair-wise different and $0 \neq u_{i} \in J_{i}, i=1,2,3$ are arbitrary elements then the pure subgroup $S=$ $=\left\{u_{1}+u_{2}, u_{2}+u_{3}\right\}_{*}^{G}$ of $G$ contains the elements $u_{1}+u_{2}, u_{1}-u_{3}=\left(u_{1}+u_{2}\right)-$ $-\left(u_{2}+u_{3}\right), u_{2}+u_{3}, u_{1}+2 u_{2}+u_{3}=\left(u_{1}+u_{2}\right)+\left(u_{2}+u_{3}\right)$ of these four types and therefore it is not completely decomposable.

b) Let the set $\left\{\hat{\tau}_{1} \cap \hat{\tau}_{2}, \hat{\tau}_{1} \cap \hat{\tau}_{3}, \hat{\tau}_{2} \cap \hat{\tau}_{3}, \hat{\tau}_{1} \cap \hat{\tau}_{2} \cap \hat{\tau}_{3}\right\}$ contains two incomparable types. Without loss of generality we can assume $\hat{\tau}_{1} \cap \hat{\tau}_{2} \| \hat{\tau}_{1} \cap \hat{\tau}_{3}$. For $\hat{\tau}_{2} \cap \hat{\tau}_{3}=$ $=\hat{\tau}_{1} \cap \hat{\tau}_{2}$ we have $\hat{\tau}_{1} \cap \hat{\tau}_{2}=\hat{\tau}_{1} \cap \hat{\tau}_{2} \cap \hat{\tau}_{3} \leqq \hat{\tau}_{1} \cap \hat{\tau}_{3}-$ a contradiction. Similarly we show that $\hat{\tau}_{2} \cap \hat{\tau}_{3}$ cannot be equal to $\hat{\tau}_{1} \cap \hat{\tau}_{3}$ so that $\hat{\tau}_{2} \cap \hat{\tau}_{3}=\hat{\tau}_{1} \cap \hat{\tau}_{2} \cap \hat{\tau}_{3}$ by the part a). Since all the $J_{i}, i=1,2,3$ are reduced there exists a prime $p$ and the elements $0 \neq u_{i} \in J_{i}, i=1,2,3$ with $h_{p}^{G}\left(u_{1}\right)=0, h_{p}^{G}\left(u_{2}\right) \geqq 1, h_{p}^{G}\left(u_{3}\right) \geqq 1$. Let us suppose that the pure subgroup $S=\left\{u_{1}+u_{2}, u_{1}+u_{3}\right\}_{*}^{G}$ of $G$ is completely decomposable, $S=I_{i}+I_{2}$. Then necessarily $\hat{\tau}\left(I_{1}\right)=\hat{\tau}_{1} \cap \hat{\tau}_{2}, \hat{\tau}\left(I_{2}\right)=\hat{\tau}_{1} \cap \hat{\tau}_{3}$ and $u_{1}+u_{2} \in I_{1}$, $u_{1}+u_{3} \in I_{2}$. Now $u_{2}-u_{3}=\left(u_{1}+u_{2}\right)-\left(u_{1}+u_{3}\right)$ yields $h_{p}^{G}\left(u_{2}-u_{3}\right)=0$ a contradiction. Therefore the set $\left\{\hat{\tau}_{1} \cap \hat{\tau}_{2}, \hat{\tau}_{1} \cap \hat{\tau}_{3}, \hat{\tau}_{2} \cap \hat{\tau}_{3}, \hat{\tau}_{1} \cap \hat{\tau}_{2} \cap \hat{\tau}_{3}\right\}$ is ordered.

c) If the set $\left\{\hat{\tau}_{1} \cap \hat{\tau}_{2}, \hat{\tau}_{1} \cap \hat{\tau}_{3}, \hat{\tau}_{2} \cap \hat{\tau}_{3}, \hat{\tau}_{1} \cap \hat{\tau}_{2} \cap \hat{\tau}_{3}\right\}$ contains two different elements then we can without loss of generality assume that $\hat{\tau}_{1} \cap \hat{\tau}_{2}>\hat{\tau}_{1} \cap \hat{\tau}_{2} \cap \hat{\tau}_{3}$. Then $\hat{\tau}_{1} \cap \hat{\tau}_{3}=\hat{\tau}_{2} \cap \hat{\tau}_{3}=\hat{\tau}_{1} \cap \hat{\tau}_{2} \cap \hat{\tau}_{3}$ because the assumption $\hat{\tau}_{1} \cap \hat{\tau}_{3}>\hat{\tau}_{1} \cap$ $\cap \hat{\tau}_{2} \cap \hat{\tau}_{3}$ (and similarly $\hat{\tau}_{2} \cap \hat{\tau}_{3}>\hat{\tau}_{1} \cap \hat{\tau}_{2} \cap \hat{\tau}_{3}$ ) leads to a contradiction $\hat{\tau}_{1} \cap \hat{\tau}_{2} \cap$ $\cap \hat{\tau}_{3}=\left(\hat{\tau}_{1} \cap \hat{\tau}_{2}\right) \cap\left(\hat{\tau}_{1} \cap \hat{\tau}_{3}\right)>\hat{\tau}_{1} \cap \hat{\tau}_{2} \cap \hat{\tau}_{3}$. Lemma 2 is therefore proved.

Lemma 3. Let $J_{i}, i=1,2,3,4,5$ be five reduced torsion free groups of rank one and of the types $\hat{\tau}_{i}, i=1,2,3,4,5$ satisfying $\hat{\tau}_{1} \cap \hat{\tau}_{2} \| \hat{\tau}_{3} \cap \hat{\tau}_{4}$ and $\hat{\tau}_{5} \geqq\left(\hat{\tau}_{1} \cap \hat{\tau}_{2}\right) \cap$ $\cap\left(\hat{\tau}_{3} \cap \hat{\tau}_{4}\right)$. Then the group $G=J_{1} \dot{+} J_{2} \dot{+} J_{3} \dot{+} J_{4} \dot{+} J_{5}$ contains a pure subgroup which is not completely decomposable.

Proof. Let $0 \neq u_{i} \in J_{i}, i=1,2,3,4,5$ be arbitrary elements. If the pure subgroup $S=\left\{u_{1}+u_{2}, u_{3}+u_{4}, u_{5}\right\}_{*}^{G}$ is completely decomposable then it suffices to use Lemma 1 for obtaining of a pure subgroup of $S$ (and hence of $G$ ) which is not completely decomposable. 
Lemma 4. (see [7]). Let $H$ be a pure subgroup of a torsion free group $G, \bar{G}=G / H$. The $\tau^{\bar{G}}(\bar{g})=\bigvee_{h \in H} \tau^{G}(g+h)$ for any $\bar{g} \in \bar{G}, \bar{g} \neq \overline{0}$.

Proof. It is clar that $\tau^{\bar{G}}(\bar{g}) \geqq \bigvee_{h \in H} \tau^{G}(g+h)$. On the other hand, if the equation $p^{k} \bar{x}=\bar{g}$ is solvable in $\bar{G}$ then $p^{k} x=g+h$ for some $h \in H$ and we are ready.

Lemma 5. Let $H=\sum_{n=1}^{\infty} J_{n}$ be a completely decomposable torsion free group of infinite countable rank and $J$ be a torsion free group of rank one such, that $\hat{\imath}(J) \| \hat{\imath}\left(J_{n}\right)$ and $\hat{\imath}\left(J_{n}\right) \geqq \hat{\imath}\left(J_{n+1}\right), n=1,2, \ldots$ Then the group $G=H+J$ contains a pure subgroup which is not completely decomposable.

Proof. For the sake of simplicity we shall write simply $\hat{\tau}, \hat{\tau}_{n}$ instead of $\hat{\tau}(J), \hat{\tau}\left(J_{n}\right)$. For the types $\hat{\tau}, \hat{\tau}_{1}, \hat{\tau}_{n}$ we can with respect to Lemma 2 assume that the types $\hat{\tau} \cap \hat{\tau}_{1}$ and $\hat{\tau}_{n}=\hat{\tau}_{1} \cap \hat{\tau}_{n}$ are comparable. Since $\hat{\tau} \geqq \hat{\tau} \cap \hat{\tau}_{1} \geqq \hat{\tau}_{n}$ for some $n$ is impossible we get $\hat{\tau} \cap \hat{\tau}_{1}<\hat{\tau}_{n}$ for all $n=1,2, \ldots$ We must consider two cases:

a) There exists a prime $p$ such that $J$ is $p$-divisible and $J_{n}$, for some $n$, is not $p$ divisible. Without loss of generality we can assume $n=1$. Let us take $0 \neq u \in J$ arbitrarily and $0 \neq u_{i} \in J_{i}$ such that $h_{p}^{G}\left(u_{i}\right)=i$. If the pure subgroup $S=\left\{u+u_{1}\right.$, $\left.u_{1}-u_{2}, u_{2}-u_{3}, \ldots, u_{n}-u_{n+1}, \ldots\right\}_{*}^{G}$ if $G$ of completely decomposable then necessarily $S^{*}\left(\hat{\tau} \cap \hat{\tau}_{1}\right)=\left\{u_{1}-u_{2}, u_{2}-u_{3}, \ldots, u_{n}-u_{n+1}, \ldots\right\}_{*}^{G}$ must be a direct summand of $S$. But any element of $S-S^{*}\left(\hat{\tau} \cap \hat{\tau}_{1}\right)$ is clearly of the type $\hat{\tau} \cap \hat{\tau}_{1}\left(=\hat{\tau} \cap \hat{\tau}_{n}\right)$, hence it is of finite $p$-height, while the factor-group $S / S^{*}\left(\hat{\imath} \cap \hat{\tau}_{1}\right)$ is $p$-divisible owing to Lemma 4 . This contradiction shows that $S$ is not completely decomposable.

b) Now we can assume that if $J$ is $p$-divisible then so is $H$. Let $p_{1}, p_{2}, \ldots$ be the sequence of all primes in increasing order of magnitude and $0 \neq u \in J$ is an arbitrary element. In this case, it is easy to see that we can choose the elements $0 \neq u_{i} \in J_{i}$ with $h_{p_{i}}^{G}\left(u_{i}\right) \geqq h_{p_{i}}^{G}(u)$. As in the part a) let us take $S=\left\{u+u_{1}, u_{1}-u_{2}, u_{2}-u_{3}, \ldots\right.$ $\left.\ldots, u_{n}-u_{n+1}, \ldots\right\}_{*}^{G}$. Any element from $S+S^{*}\left(\hat{\tau} \cap \hat{\tau}_{1}\right)$ is of the type $\hat{\tau} \cap \hat{\tau}_{1}$ while the factor-group $S / S^{*}\left(\hat{\tau} \cap \hat{\tau}_{1}\right)$ is of the type $\hat{t}^{\prime} \geqq \hat{\tau}$ by Lemma 4 . The same arguments as in the part a) show that $S$ is not completely decomposable.

Lemma 6. Let $\hat{\tau}, \hat{\tau}^{\prime}$ be two incomparable types and $J$ be a torsion free group of rank one and of the type $\tau$. Further, let $H=\sum_{n=1}^{\infty} J_{n}$ be a completely decomposable torsion free group of infinite countable rank where $\hat{\tau}\left(J_{n}\right)=\hat{\tau}_{n} \geqq \hat{\tau}^{\prime}$ and $\hat{\tau}_{n} \cap \hat{\tau}=$ $=\hat{\tau}^{\prime} \cap \hat{\tau}$ for all $n=1,2, \ldots$ Then $G=H+J$ contains a pure subgroup which is not completely decomposable.

Proof. For any $n=1,2, \ldots$ there is $\hat{\tau} \| \hat{\tau}_{n}$ since the assumption $\hat{\tau} \geqq \hat{\tau}_{n}$ leads to a contradiction $\hat{\tau} \geqq \hat{\tau}^{\prime} \cap \hat{\tau}=\hat{\tau}_{n} \cap \hat{\tau}=\hat{\tau}_{n} \geqq \hat{\tau}^{\prime}$ while the assumption $\hat{\tau}_{n} \geqq \hat{\tau}$ leads to 
a contradiction $\hat{\tau}^{\prime} \geqq \hat{\tau} \cap \hat{\tau}=\hat{\tau}_{n} \cap \hat{\tau}=\hat{\tau}$. As in the preceding proof we shall consider two cases:

a) There exists a prime $p$ such that $J$ is $p$-divisible and $H$ is not $p$-divisible. Taking $0 \neq u \in J$ arbitrarily and $0 \neq u_{i} \in J_{i}$ such that $h_{p}^{G}\left(u_{i}\right)=i$ we easily obtain that the group $S=\left\{u+u_{1}, u_{1}-u_{2}, u_{2}-u_{3}, \ldots, u_{n}-u_{n+1}, \ldots\right\}_{*}^{G}$ contains only the elements the types of which are either greater than or equal to $\hat{\tau}^{\prime}$ or equal to $\hat{\tau} \cap \hat{\tau}^{\prime}$. If $S$ is completely decomposable then $S\left(\hat{\tau}^{\prime}\right)$ is a direct summand of $S$. But any element of $S-S\left(\hat{\tau}^{\prime}\right)$ is of the type $\hat{\tau} \cap \hat{\tau}^{\prime}$, hence it is of finite $p$-height, while the factor-group $S / S\left(\hat{\imath}^{\prime}\right)$ is $p$-divisible owing to Lemma 4 . This contradiction shows that $S$ is not completely decomposable.

b) We shall assume now that if $J$ is $p$-divisible then so is $H$. We omit the proof of this part since it is similar to the proof of the corresponding part of the preceding Lemma.

Lemma 7. Let $G$ be a completely decomposable torsion free group having the property that for any four not necessarily different elements $\hat{\tau}_{i}$, $i=1,2,3,4$ from $T(G)$ with $\hat{\tau}_{1} \cap \hat{\tau}_{2} \| \hat{\tau}_{3} \cap \hat{\tau}_{4}$ there is $G\left(\hat{\tau}_{1} \cap \hat{\tau}_{2}\right) \cap G\left(\hat{\tau}_{3} \cap \hat{\tau}_{4}\right)=0$. Then for any three elements $\hat{\tau}_{1}, \hat{\tau}_{2}, \hat{\tau}_{3}$ from $T(G)$ the set $\left\{\tau_{1} \cap \tau_{2}, \tau_{1} \cap \tau_{3}, \hat{\tau}_{2} \cap \tau_{3}, \tau_{1} \cap \tau_{2} \cap\right.$ $\left.\cap \tau_{3}\right\}$ is ordered and contains at most two different elements.

Proof. If the considered set is not ordered then we can without loss of generality assume that $\hat{\tau}_{1} \cap \hat{\tau}_{2} \| \hat{\tau}_{1} \cap \hat{\tau}_{3}$ which implies $0 \neq G\left(\hat{\tau}_{1}\right) \subseteq G\left(\hat{\tau}_{1} \cap \hat{\tau}_{2}\right) \cap G\left(\hat{\tau}_{1} \cap \hat{\tau}_{3}\right)-$ a contradiction. Now if in the considered set is $\hat{\tau}_{1} \cap \hat{\tau}_{2}>\hat{\tau}_{1} \cap \hat{\tau}_{2} \cap \hat{\tau}_{3}$ then necessarily $\hat{\tau}_{1} \cap \hat{\tau}_{3}=\hat{\tau}_{2} \cap \hat{\tau}_{3}=\hat{\tau}_{1} \cap \hat{\tau}_{2} \cap \hat{\tau}_{3}$ (see the part c) of the proof of Lemma 2).

Lemma 8. Let $G$ be a completely decomposable torsion free group satisfying the condition of the preceding Lemma. If $\hat{\tau}_{n}^{\prime}, \hat{\tau}_{n}^{\prime \prime}$ are elements of $T(G)$ such that $\hat{\tau}_{n}^{\prime} \cap$ $\cap \hat{\tau}_{n}^{\prime \prime}=\hat{\tau}_{n}$ and $\hat{\tau}_{1}>\hat{\tau}_{2}>\ldots$ then for any $n=1,2, \ldots$ there is either $\hat{\tau}_{n}=\hat{\tau}_{1}^{\prime} \cap \hat{\tau}_{n}^{\prime}$ or $\hat{\tau}_{n}=\hat{\tau}_{1}^{\prime} \cap \hat{\tau}_{n}^{\prime \prime}$.

Proof. For $n=1$ there is nothing to prove. For $n>1$ we have $\hat{\tau}_{1}^{\prime} \geqq \hat{\tau}_{1} \cap \hat{\tau}_{n}$, $\hat{\tau}_{n}^{\prime} \geqq \hat{\tau}_{n}$ and hence $\hat{\tau}_{1}^{\prime} \cap \hat{\tau}_{n}^{\prime} \geqq \hat{\tau}_{n}$. For $\hat{\tau}_{1}^{\prime} \cap \hat{\tau}_{n}^{\prime}>\hat{\tau}_{n}$ Lemma 7 gives $\hat{\tau}_{1}^{\prime} \cap \hat{\tau}_{n}^{\prime}>\hat{\tau}_{n}=$ $=\hat{\tau}_{n}^{\prime} \cap \hat{\tau}_{n}^{\prime \prime}=\hat{\tau}_{1}^{\prime} \cap \hat{\tau}_{n}^{\prime \prime}$ and we are ready.

Lemma 9. Let $G=J+\sum_{n=1}^{\infty} J_{n}$ be a completely decomposable torsion free group satisfying the condition of Lemma 7 where $J, J_{n}, n=1,2, \ldots$ are of rank one and of the types $\hat{\tau}(J)=\hat{\tau}, \hat{\tau}\left(J_{n}\right)=\hat{\tau}_{n}^{\prime}$ and $\hat{\tau} \cap \hat{\tau}_{n}^{\prime}=\hat{\tau}_{n}, \hat{\tau}_{n}>\tau_{n+1}, n=1,2, \ldots$ If $S$ is a rank one pure subgroup of $G$ such that $S \cap J=0, S \cap J_{n}=0, n=1,2, \ldots$ then $\hat{\imath}(S)=\hat{\tau}_{n}$ for some $n$. 
Proof. If an element $0 \neq s \in S$ has a non-zero component in $J, s=u+\sum_{i=1}^{k} u_{i}$, $0 \neq u \in J, \quad 0 \neq u_{i} \in J_{n_{i}}, \quad n_{1}<n_{2}<\ldots<n_{k}, \quad k \geqq 1 \quad$ then $\hat{\tau}^{G}(s)=\hat{\tau} \cap \bigcap_{i=1} \hat{\tau}_{n_{i}}^{\prime}=$ $=\bigcap_{i=1}^{k} \hat{\tau}_{n_{i}}=\hat{\tau}_{n_{k}}$. If the component of $s$ in $J$ is zero, $s=\sum_{i=1}^{k} u_{i}, 0 \neq u_{i} \in J_{n_{i}}, n_{1}<$ $<n_{2}<\ldots<n_{k}, k \geqq 2$ then $\hat{\tau} \cap \hat{\tau}_{n_{k-1}}^{\prime}>\hat{\tau} \cap \hat{\tau}_{n_{k}}^{\prime}=\hat{\tau}_{n_{k}}$ yields $\hat{\tau}_{n_{k-1}}^{\prime} \cap \hat{\tau}_{n_{k}}^{\prime}=\hat{\tau} \cap$ $\cap \hat{\tau}_{n_{k}}^{\prime}$ by Lemma 7 and $\hat{\tau}^{G}(s)=\hat{\tau}_{n_{1}}^{\prime} \cap \hat{\tau}_{n_{2}}^{\prime} \cap \ldots \cap \hat{\tau}_{n_{k-1}}^{\prime} \cap \hat{\tau}_{n_{k}}^{\prime}=\hat{\tau}_{n_{1}}^{\prime} \cap \hat{\tau}_{n_{2}}^{\prime} \cap \ldots$ $\ldots \cap \hat{\tau}_{n_{k-2}}^{\prime} \cap \hat{\tau} \cap \hat{\tau}_{n_{k}}^{\prime}=\hat{\tau}_{n_{1}} \cap \hat{\tau}_{n_{2}} \cap \ldots \cap \hat{\tau}_{n_{k-2}} \cap \hat{\tau}_{n_{k}}=\hat{\tau}_{n_{k}}$.

Lemma 10. Let $G$ be a completely decomposable torsion free group having the property that for any four not necessarily different elements $\hat{\tau}_{i}, i=1,2,3,4$ from $T(G)$ with $\hat{\tau}_{1} \cap \hat{\tau}_{2} \| \hat{\tau}_{3} \cap \hat{\tau}_{4}$ there is $G\left(\hat{\tau}_{1} \cap \hat{\tau}_{2}\right) \cap G\left(\hat{\tau}_{3} \cap \hat{\tau}_{4}\right)=0$. Then any finite set $\hat{\tau}_{1}, \hat{\tau}_{2}, \ldots, \hat{\tau}_{n}$ of elements of $T(G)$ contain two elements having the same intersection as this set.

Proof. We shall use the induction by $n$. For $n=3$ our assertion follows immediately from Lemma 7. Let us suppose $n>3$ and our assertion is true for $n-1$. By the induction hypothesis we can without loss of generality assume $\hat{\tau}_{1} \cap \hat{\tau}_{2} \cap \ldots$ $\ldots \cap \hat{\tau}_{n-1}=\hat{\tau}_{1} \cap \hat{\tau}_{2}$ hence $\hat{\tau}_{1} \cap \hat{\tau}_{2} \cap \ldots \cap \hat{\tau}_{n}=\hat{\tau}_{1} \cap \hat{\tau}_{2} \cap \hat{\tau}_{n}$ and our lemma follows by the induction hypothesis.

Lemma 11. Under the hypothesis of the preceding lemma the set $\left\{\hat{\tau}^{\prime}, \hat{\tau}^{\prime} \in \hat{\tau}(G)\right.$, $\left.\hat{\tau}^{\prime}<\hat{\tau}\right\}$ where $\hat{\tau}$ is an arbitrary element of $\hat{\tau}(G)$ contains at most one maximal element.

Proof: Suppose that $\hat{\tau}_{1}, \hat{\tau}_{2}$ are two maximal elements of the considered set. Then necessarily $\hat{\tau}_{1} \| \hat{\tau}_{2}$ and the inclusion $0 \neq G(\hat{\tau}) \subseteq G\left(\hat{\tau}_{1}\right) \cap G\left(\hat{\tau}_{2}\right)$ together with the preceding lemma yields a contradiction.

Lemma 12. Let $G=\sum_{\alpha \in A} J_{\alpha}$ be a completely decomposable torsion free group where $J_{\alpha}$ is of rank one and of the type $\hat{\tau}_{\alpha}$. If $A$ is uncountable and for any $\alpha, \beta \in A$, $\alpha \neq \beta$ there is $\hat{\tau}_{\alpha} \| \hat{\tau}_{\beta}$ and $\hat{\tau}_{\alpha} \cap \hat{\tau}_{\beta}=\hat{\tau}$ then $G$ contains a pure subgroup which is not completely decomposable.

Proof. Let $B$ be any infinite countable subset of $A$. We can clearly assume that $B$ is the set of all ordered couples $(i, j)$ of natural integers. Hence $H=\sum_{\alpha \in B} J_{\alpha}=\sum_{i, j=1}^{\infty} J_{i j}$ is a countable direct summand of $G$. If $p_{1}, p_{2}, \ldots$ is the sequence of all primes in increasing order of magnitude then we can choose $0 \neq u_{i j} \in J_{i j}, i, j=1,2, \ldots$ with $h_{p_{i}}^{G}\left(u_{i j}\right) \geqq j$. Let $J$ be one of the $J_{\alpha}, \alpha \notin B$ and $0 \neq u \in J$ an arbitrary element. Taking $0 \neq u_{\alpha} \in J_{\alpha}, J_{\alpha} \neq J, \alpha \notin B$ arbitrarily let us put $S=\left\{u-u_{\alpha}, u-u_{i j}\right.$, 
$\alpha \in A-B, i, j=1,2, \ldots\}_{*}^{G}$. Supposing $S$ is completely decomposable we get that $\left\{u-u_{i j}, i, j=1,2, \ldots\right\}_{*}^{G}$ is contained in a countable completely decomposable direct summand $S^{\prime}$ of $S$. On the one hand the factor group $S / S^{\prime}$ is homogeneous of the type $\hat{\tau}$ since so is $S$. On the other hand from the uncountability of $S$ it follows the existence of $u-u_{\alpha} \notin S^{\prime}$. Further, if $k_{i}$ is a natural integer, $k_{i}<h_{p_{i}}^{G}\left(u_{\alpha}\right)+1$ (here we put $\infty+1=\infty)$ then $h_{p_{i}}^{G}\left(u_{\alpha}-u_{i, k_{i}}\right)=h_{p_{i}}^{G}\left(\left(u-u_{i, k_{i}}\right)-\left(u-u_{\alpha}\right)\right) \geqq k_{i}$. Therefore, by Lemma 4 , the coset $\left(u-u_{\alpha}\right)+S^{\prime}$ is at least of the type $\hat{\tau}_{\alpha}>\hat{\tau}-$ a contradiction proving our lemma.

Lemma 13. Let $G$ be a completely decomposable torsion free group of finite rank $n, G=J_{1}+J_{2}+\ldots+J_{n}$ and let $S$ be a pure subgroup of $G$ of rank $r<n$. Then $S$ contains a maximal linearly independent set $s_{1}, s_{2}, \ldots, s_{r}$ of the form

$$
\begin{aligned}
& s_{1}=x_{1} \quad+\sum_{i=r+1}^{n} \lambda_{1, i} x_{i}, \\
& s_{2}=x_{2} \quad+\sum_{i=r+1}^{n} \lambda_{2, i} x_{i}, \\
& \ldots \ldots \ldots \ldots \ldots \ldots \ldots \ldots \ldots \ldots \ldots \ldots \\
& s_{r}=\quad x_{r}+\sum_{i=r+1}^{n} \lambda_{r, i} x_{i},
\end{aligned}
$$

where $x_{i} \in J_{i}$ in a suitable enumeration of $J_{i}$ 's.

Proof. We shall use the induction by $r$. For $r=1$ there is nothing to prove. For $r>1$ we can assume that $0 \neq s_{1}^{\prime}=x_{1}^{\prime}+\sum_{i=2}^{n} \lambda_{i}^{\prime} x_{i}^{\prime}, x_{i}^{\prime} \in J_{i}, i=1,2, \ldots, n, x_{1}^{\prime} \neq 0$ is an arbitrary element of $S$. The group $S \cap\left(\sum_{n}^{n} J_{i=2}\right)$ is of rank $r-1$ since the factorgroup $S / S \cap\left(\sum_{i=2}^{n} J_{i}\right) \cong\left\{S \sum_{i=2}^{n} J_{i}\right\} / \dot{\sum}_{i=2}^{n} J_{i}$ is non-zero of rank one. By the induction hypothesis it clearly contains a maximal linearly independent set $s_{2}^{\prime}, \ldots, s_{r}^{\prime}$ of the form $\left(J_{2}, \ldots, J_{n}\right.$ are suitably enumerated)

$$
\begin{aligned}
& s_{2}^{\prime}=x_{2}^{\prime \prime} \quad+\sum_{i=r+1}^{n} \lambda_{2, i}^{\prime} x_{i}^{\prime \prime}, \\
& \cdots \ldots \ldots \ldots \ldots \cdots \cdots \cdots \cdots \cdots \cdots \\
& s_{r}^{\prime}=x_{r}^{\prime \prime}+\sum_{i=r+1}^{n} \lambda_{r, i}^{\prime} x_{i}^{\prime \prime} .
\end{aligned}
$$

Now it is easy to see that if we add a suitable linear combination of $s_{2}^{\prime}, \ldots, s_{r}^{\prime}$ to a suitable multiple of $s_{1}^{\prime}$ (rewritten in a new enumeration of $J_{2}, \ldots, J_{n}$ ) we get a maximal linearly independent set of $S$ having the form (1). 
Lemma 14. Let $G_{1}=\sum_{i=1}^{n_{1}} J_{i}$ and $G_{2}=\sum_{j=1}^{n_{2}} I_{j}$ be completely decomposable torsion free groups of finite ranks $n_{1}, n_{2}$ resp. If $S$ is a pure subgroup of $G=G_{1}+G_{2}$ of rank $r<n_{1}+n_{2}, r\left(S \cap G_{i}\right)=r_{i}, i=1,2, r=r_{1}+r_{2}+r^{\prime}$ then $S$ contains a maximal linearly independent set $s_{1}, s_{2}, \ldots, s_{r_{1}}, t_{1}, t_{2}, \ldots, t_{r_{2}}, u_{1}, u_{2}, \ldots, u_{r^{\prime}}$ of the form

$$
\begin{array}{lll}
s_{1}=x_{1} & & +\sum_{i=r_{1}+1}^{n_{1}} \lambda_{1, i} x_{i},
\end{array}
$$

where $x_{i} \in J_{i}, y_{j} \in I_{j}$ in a suitable enumeration of the groups $J_{i}$ and $I_{j}$.

Proof. We shall use the induction by $r^{\prime}$. For $r^{\prime}=0$ it suffices to use Lemma 13 for $S \cap G_{i}, i=1,2$. For $r^{\prime}>0$ let $S^{\prime}$ be any pure subgroup of $S$ of rank $r-1$ containing $S \cap G_{1}, S \cap G_{2}$. By the induction hypothesis $S^{\prime}$ contains a maximal linearly independent set $s_{1}^{\prime}, s_{2}^{\prime}, \ldots, s_{r_{1}}^{\prime}, t_{1}^{\prime}, t_{2}^{\prime}, \ldots, t_{r_{2}}^{\prime}, u_{1}^{\prime}, u_{2}^{\prime}, \ldots, u_{r^{\prime}-1}^{\prime}$ having the form like (2) (where instead of $x_{i}, y_{j}$ are $x_{i}^{\prime}, y_{j}^{\prime}$ etc.). It is easy to see that one can choose an element $u_{r^{\prime}}^{\prime}$ in $S-S^{\prime}$ having the form $u_{r^{\prime}}^{\prime}=\sum_{i=r_{1}+r^{\prime}}^{n_{1}} v_{r^{\prime}, i} x_{i}^{\prime}+\sum_{i=r_{2}+1}^{n_{2}} x_{r^{\prime}, i}^{\prime} y_{i}^{\prime}$. Here not all $v_{r^{\prime}, i}^{\prime}$ are equal to zero since for $v_{r^{\prime}, i}^{\prime}=0, i=r_{1}+r^{\prime}, \ldots, n_{1}$ the element $u_{r^{\prime}}^{\prime}$, lies in $S \cap G_{2}$ and therefore in $S^{\prime}$. Now the proof can be easily finished. 
Lemma 15. Let

$$
\boldsymbol{A}=\left(\begin{array}{c}
a_{11}, a_{12}, \ldots, a_{1 n} \\
a_{21}, a_{22}, \ldots, a_{2 n} \\
\ldots \ldots \ldots, \ldots . \\
a_{n 1}, a_{n 2}, \ldots, a_{n n}
\end{array}\right)
$$

be a regular matrix over the ring of integers and let us denote its determinant by $D$. If for some integers $\gamma_{1}, \gamma_{2}, \ldots, \gamma_{n}$ a prime $p$ divides all the $\sum_{i=1}^{n} \gamma_{i} a_{i j}, j=1$, $2, \ldots, n$ then $p$ divides all the $\gamma_{i} D, i=1,2, \ldots, n$.

Proof. Considering a row vector $\gamma=\left(\gamma_{1}, \gamma_{2}, \ldots, \gamma_{n}\right)$ as a $(1, n)$-matrix we can multiply the matrix product $\boldsymbol{\gamma}$. $\boldsymbol{A}$ by the adjoint matrix of $\boldsymbol{A}$ on the right from which the assertion easily follows.

Lemma 16. Let $G_{1}=\sum_{i=1}^{n_{1}} J_{i}$ and $G_{2}=\sum_{j=1}^{n_{2}} I_{j}$ be completely decomposable torsion free groups of finite ranks $n_{1}, n_{2}$ respectively satisfying $\hat{\tau}_{1} \cap \hat{\tau}_{2}=\hat{\tau}$ for any $\hat{\tau}_{i} \in \hat{\tau}\left(G_{i}\right), i=1$, 2. Let $S, S^{\prime}$ be pure subgroups of $G=G_{1}+G_{2}$ such that $\left(S \cap G_{1} \dot{+}\right.$ $\left.+S \cap G_{2}\right) \subseteq S^{\prime} \subseteq S$. Then the factor-group $S / S^{\prime}$ is homogeneous of the type $\hat{\tau}$.

Proof. If we denote $r\left(S \cap G_{i}\right)=r_{i}, i=1,2, r\left(S^{\prime}\right)=r, r=r_{1}+r_{2}+r^{\prime}$, then by Lemma $14 S^{\prime}$ contains a maximal linearly independent set of the form (2). Any non-zero element from $S-S^{\prime}$ has a non-zero multiple which is a linear combination of the elements $x_{1}, x_{2}, \ldots, x_{n_{1}}, y_{1}, y_{2}, \ldots, y_{n_{2}}$ and the corresponding coset of $S / S^{\prime}$ clearly contains an element $s \neq 0$ of the form

$$
s=\sum_{i=\boldsymbol{r}_{1}+\boldsymbol{r}^{\prime}+1}^{n_{1}} \varrho_{i} x_{i}+\sum_{i=r_{2}+1}^{n_{2}} \sigma_{i} y_{i},
$$

where not all $\varrho_{i}$ and not all $\sigma_{i}$ are equal to zero. From the linear independence of $s_{1}, s_{2}, \ldots, s_{r_{1}}, t_{1}, t_{2}, \ldots, t_{r_{2}}, u_{1}, u_{2}, \ldots, u_{r^{\prime}}, s$ it immediately follows the linear independence of the elements $\sum_{i=r_{2}+1}^{n_{2}} x_{1, i} y_{i}, \sum_{i=r_{2}+1}^{n_{2}} x_{2, i} y_{i}, \ldots, \sum_{i=r_{2}+1}^{n_{2}} x_{r^{\prime} i} y_{i}, \sum_{i=r_{2}+1}^{n_{2}} \sigma_{i} y_{i}$ and therefore the vectors $x_{j}=\left(x_{j, r_{2}+1}, \ldots, x_{j, n_{2}}\right), j=1,2, \ldots, r$, and $\sigma=\left(\sigma_{r_{2}+1}, \ldots, \sigma_{n_{2}}\right)$ are linearly independent as rational vectors. Without loss of generality (taking a suitable enumeration of $y$ 's) we can assume that the determinant

$$
D=\left|\begin{array}{llll}
x_{1, r_{2}+1}, & x_{1, r_{2}+2}, & \ldots, & x_{1, r_{2}+r^{\prime}+1} \\
x_{2, r_{2}+1}, & x_{2, r_{2}+2}, & \ldots, & x_{2, r_{2}+r^{\prime}+1} \\
\vdots & \vdots & & \vdots \\
x_{r^{\prime}, r_{2}+1}, & x_{r^{\prime}, r_{2}+2}, & \ldots, & x_{r^{\prime}, r_{2}+r^{\prime}+1} \\
\sigma_{r_{2}+1}, & \sigma_{r_{2}+2}, & \ldots, & \sigma_{r_{2}+r^{\prime}+1}
\end{array}\right|
$$


is non-zero. Now for an arbitrary element $s^{\prime} \in S^{\prime}$ there exist the integers $\alpha, \alpha_{1}, \alpha_{2}, \ldots$ $\ldots, \alpha_{\boldsymbol{r}_{1}}, \beta_{1}, \beta_{2}, \ldots, \beta_{r_{2}}, \gamma_{\boldsymbol{r}_{1}+1}, \ldots, \gamma_{\boldsymbol{r}_{1}+\boldsymbol{r}^{\prime}}$ with $\alpha \neq 0$ and $\alpha s^{\prime}=\sum_{i=1}^{r_{1}} \alpha_{i} s_{i}+\sum_{i=1}^{r_{2}} \beta_{i} t_{i}+$ $+\sum_{i=1}^{r^{\prime}} \gamma_{r_{1}+i} u_{i}$. Using (2) and (3) one easily get

$$
\begin{aligned}
\alpha\left(s+s^{\prime}\right)= & \sum_{i=1}^{r_{1}} \alpha_{i} x_{i}+\sum_{i=r_{1}+1}^{r_{1}+r^{\prime}}\left(\sum_{j=1}^{r_{1}} \alpha_{j} \lambda_{j i}+\gamma_{i}\right) x_{i}+ \\
& +\sum_{i=r_{1}+r^{\prime}+1}^{n_{1}}\left(\sum_{j=1}^{r_{1}} \alpha_{j} \lambda_{j i}+\sum_{j=1}^{r^{\prime}} \gamma_{r_{1}+j^{\prime}} v_{j i}+\alpha \varrho_{i}\right) x_{i}+ \\
& +\sum_{i=1}^{r_{2}} \beta_{i} y_{i}+\sum_{i=r_{2}+1}^{n_{2}}\left(\sum_{j=1}^{r_{2}} \beta_{j} \mu_{j i}+\sum_{j=1}^{r^{\prime}} \gamma_{r_{1}+j} \chi_{j i}+\alpha \sigma_{i}\right) y_{i} .
\end{aligned}
$$

From the fact that any type from $\hat{\tau}\left(G_{1}\right)$ has with any type from $\hat{\tau}\left(G_{2}\right)$ the same intersection $\hat{\tau}$ we conclude that for almost all primes $p$ and all $i=1,2, \ldots, n ; j=1,2, \ldots$ $\ldots, n$ all the $\min \left(h_{p}^{G}\left(x_{i}\right), h_{p}^{G}\left(y_{j}\right)\right)$ are equal to the same $k$ (depending on $p$, of course), where $k$ is either a natural integer, or 0 or $\infty$. If $p$ is such a prime then either all $h_{p}^{G}\left(x_{i}\right), i=1,2, \ldots, n_{1}$ or all $h_{p}^{G}\left(y_{j}\right), j=1,2, \ldots, n_{2}$ are equal to $k$. For such a prime $p$ which moreover does not divide $D$ and all non-zero $\varrho_{i}, \sigma_{j}, i=r_{1}+r^{\prime}+1, \ldots, n_{1}$, $j=r_{2}+1, \ldots, n_{2}$ we now consider two cases.

a) $h_{p}^{G}\left(x_{1}\right)=h_{p}^{G}\left(x_{2}\right)=\ldots=h_{p}^{G}\left(x_{n_{1}}\right)=k \neq \infty$. If $h_{p}^{G}\left(\alpha\left(s+s^{\prime}\right)\right)=k+l$ then from (4) one get $p^{l} \mid \alpha_{i}, i=1,2, \ldots, r_{1}$, hence $p^{l} \mid \gamma_{r_{1}+i}, i=1,2, \ldots, r^{\prime}$ and finally $p^{l} \mid \alpha \varrho_{i}$ and $p^{l} \mid \alpha$. Therefore $h_{p}^{G}\left(s+s^{\prime}\right) \leqq k$.

b) $h_{p}^{G}\left(y_{1}\right)=h_{p}^{G}\left(y_{2}\right)=\ldots=h_{p}^{G}\left(y_{n_{2}}\right)=k \neq \infty$. If $h_{p}^{G}\left(\alpha\left(s+s^{\prime}\right)=k+l\right.$ then $p^{l} \mid \beta_{i}, i=1,2, \ldots, r_{2}$. Hence $p^{l} \mid\left(\sum_{j=1}^{r^{\prime}} \gamma_{r_{1}+j} \chi_{j i}+\alpha \sigma_{i}\right), i=r_{2}+1, \ldots, n_{2}$. By Lemma $15 p^{l} \mid \alpha D$ and $p^{l} \mid \alpha$. Therefore $h_{p}^{G}\left(s+s^{\prime}\right) \leqq k$, again.

Let $p$ be a prime from the remaining set of primes. If the component of $\tau$ at the prime $p$ is finite then either all $h_{p}^{G}\left(x_{i}\right), i=1,2, \ldots, n_{1}$ or all $h_{p}^{G}\left(y_{j}\right), j=1,2, \ldots, n_{2}$ are finite. We consider these two cases separately.

a) All the $h_{p}^{G}\left(x_{i}\right), i=1,2, \ldots, n_{1}$ are finite. Let $k$ be the greatest element among the $h_{p}^{G}\left(x_{i}\right)$ and $l$ the maximal exponent for which $p^{l}$ divides some of non-zero integers $\varrho_{i}, \sigma_{j}, i=r_{1}+r^{\prime}+1, \ldots, n_{1}, j=r_{2}+1, \ldots, n_{2}$. If $h_{p}^{G}\left(\alpha\left(s+s^{\prime}\right)\right)=k+l+m$ then $p^{l+m} \mid \alpha_{i}, i=1,2, \ldots, r_{1}$ hence $p^{l+m} \mid \gamma_{r_{1}+i}, \quad i=1,2, \ldots, r^{\prime}$ and $p^{l+m} \mid \alpha \varrho_{i}$. Therefore $p^{m} \mid \alpha$ and $h_{p}^{G}\left(s+s^{\prime}\right) \leqq k+l$.

$\beta$ ) All the $h_{p}^{G}\left(y_{j}\right), j=1,2, \ldots, n_{2}$ are finite. If $k$ is the greatest element among the $h_{p}^{G}\left(y_{j}\right)$ and $l$ is the greatest exponent for which $p^{l} \mid D$ then for $h_{p}^{G}\left(\alpha\left(s+s^{\prime}\right)=k+\right.$ $+l+m$ we get $p^{l+m} \mid \beta_{i}, i=1,2, \ldots, r_{2}$, hence $p^{l+m} \mid\left(\sum_{j=1}^{r^{\prime}} \gamma_{r_{1}+j} \chi_{j i}+\alpha \sigma_{i}\right), i=$ $=r_{2}+1, \ldots, n_{2}$ so that $p^{l+m} \mid \alpha D$ by Lemma 15 and $p^{m} \mid \alpha$. Therefore $h_{p}^{G}\left(s+s^{\prime}\right) \leqq$ $\leqq k+l$. 
Finally, if the component of $\tau$ at the prime $p$ is infinite then $S$ is clearly $p$-divisible and hence $S / S^{\prime}$ is $p$-divisible, two. To finish the proof of Lemma 16 it suffices now to apply Lemma 4.

Lemma 17. Let $G_{1}=\sum_{i=1}^{n_{1}} J_{i}$ and $G_{2}=\sum_{j=1}^{n_{2}} I_{j}$ be completely decomposable torsion free groups of finite ranks $n_{1}, n_{2}$ respectively satisfying $\hat{\tau}_{1} \cap \hat{\tau}_{2}=\hat{\tau}$ for any $\hat{\tau}_{i} \in \hat{\tau}\left(G_{i}\right), i=1$, 2. If $S$ is a pure subgroup of $G=G_{1}+G_{2}$ then $S$ is a direct sum of $\left(S \cap G_{1}\right)+\left(S \cap G_{2}\right)$ and a completely decomposable group.

Proof. It suffices to use Lemma 16 and Theorem 46.4 from [1].

In the following it will be useful this simple consequence of Theorem 1 from J. S. P. Wang's paper [2].

Wang's theorem. Let a torsion free group $G$ be of the form $G=G_{1}+G_{2}$ where $G_{2}$ is completely decomposable with inversely well-ordered type set and for any $\hat{\tau}_{i} \in \hat{\tau}\left(G_{i}\right), i=1,2$ there is $\hat{\tau}_{1} \geqq \hat{\tau}_{2}$. Then any pure subgroup $S$ of $G$ is a direct sum of $S \cap G_{1}$ and a completely decomposable group.

Now we are ready to prove the main result.

Theorem 1. A completely decomposable torsion free group $G$ the type set of which satisfies the maximum condition has the property that any its pure subgroup is completely decomposable if and only if $G=D+H$ where $D$ is divisible and $H$ reduced and

a) for any four not necessarily different elements $\hat{\tau}_{i}, i=1,2,3,4$ from $T(H)$ with $\hat{\tau}_{1} \cap \hat{\tau}_{2} \| \hat{\tau}_{3} \cap \hat{\tau}_{4}$ there is $H\left(\hat{\tau}_{1} \cap \hat{\tau}_{2}\right) \cap H\left(\hat{\tau}_{3} \cap \hat{\tau}_{4}\right)=0$,

b) for any two incomparable elements $\hat{\tau}^{\prime}, \hat{\tau}^{\prime \prime}$ from $T(H)$ the set $\left\{\hat{\tau}_{1} \cap \hat{\tau}_{2}, \hat{\tau}_{i} \in T(H)\right.$, $\left.\hat{\tau}_{i} \geqq \hat{\tau}^{\prime} \cap \hat{\tau}^{\prime \prime}, i=1,2\right\}$ satisfies the minimum condition,

c) for any three not necessarily different elements $\hat{\tau}_{1}, \hat{\tau}_{2}, \hat{\tau}_{3}$ from $T(H)$ with $\hat{\tau}_{3} \| \hat{\tau}_{1} \cap \hat{\tau}_{2}$ the subgroup $H\left(\hat{\tau}_{1} \cap \hat{\tau}_{2}\right)$ is of finite rank,

d) the set of all maximal elements of $T(H)$ is at most countable.

Proof. 1) Necessity: If the completely decomposable group $G=D+H$ has the property that any its pure subgroup is completely decomposable then $H$ has the same property.

Condition a): We must consider three cases.

$\alpha$ ) If $\hat{\tau}_{1} \| \hat{\tau}_{2}$ are from $T(H)$, then $H\left(\hat{\tau}_{1}\right) \cap H\left(\hat{\tau}_{2}\right)=0$ by Lemma 1 .

$\beta$ ) Let $\hat{\tau}_{1}, \hat{\tau}_{2}, \hat{\tau}_{3}$ be different elements from $T(H), \hat{\tau}_{1} \cap \hat{\tau}_{2} \| \hat{\tau}_{3}$. Let us suppose one of the $\hat{\tau}_{1}, \hat{\tau}_{2}$, say $\hat{\tau}_{1}$, is greater than or equal to $\hat{\tau}_{3}$. Then $\hat{\tau}_{3}=\hat{\tau}_{1} \cap \hat{\tau}_{3}>\hat{\tau}_{1} \cap \hat{\tau}_{2} \cap$ $\cap \hat{\tau}_{3}=\hat{\tau}_{1} \cap \hat{\tau}_{2}$ by Lemma 2, which contradicts to $\hat{\tau}_{1} \cap \hat{\tau}_{2} \| \hat{\tau}_{3}$. Hence no of the $\hat{\tau}_{1}, \hat{\tau}_{2}$ is greater than $\left(\hat{\tau}_{1} \cap \hat{\tau}_{2}\right) \vee \hat{\tau}_{3}$ and it suffices to use Lemma 1 . 
$\gamma$ ) Let the types $\hat{\tau}_{1}, \hat{\tau}_{2}, \hat{\tau}_{3}, \hat{\tau}_{4}$, be different elements from $T(H), \hat{\tau}_{1} \cap \hat{\tau}_{2} \| \hat{\tau}_{3} \cap \hat{\tau}_{4}$. Let us suppose that one of the $\hat{\tau}_{i}$, say $\hat{\tau}_{1}$ is greater than or equal to $\left(\hat{\tau}_{1} \cap \hat{\tau}_{2}\right) \vee$ $\vee\left(\hat{\tau}_{3} \cap \hat{\tau}_{4}\right)$. Taking arbitrarily the non-zero elements $u_{3} \in J_{3}, u_{4} \in J_{4}$ and denoting $J_{5}=\left\{u_{3}+u_{4}\right\}_{*}^{G}$ we get $\hat{\tau}\left(J_{5}\right)=\hat{\tau}_{5}=\hat{\tau}_{3} \cap \hat{\tau}_{4}$. For the pure subgroup $S=J_{1} \dot{+}$ $+J_{2}+J_{5}$ of $H$ it holds $\hat{\tau}_{1} \cap \hat{\tau}_{5}>\hat{\tau}_{1} \cap \hat{\tau}_{2}=\hat{\tau}_{2} \cap \hat{\tau}_{5}=\hat{\tau}_{1} \cap \hat{\tau}_{2} \cap \hat{\tau}_{5}$ owing to the hypothesis and Lemma 2. But then the inequality $\hat{\tau}_{1} \cap \hat{\tau}_{5}=\hat{\tau}_{1} \cap \hat{\tau}_{3} \cap \hat{\tau}_{4}=\hat{\tau}_{3} \cap$ $\cap \hat{\tau}_{4}>\hat{\tau}_{1} \cap \hat{\tau}_{2}$ contradicts to the incomparability of $\hat{\tau}_{1} \cap \hat{\tau}_{2}$ and $\hat{\tau}_{3} \cap \hat{\tau}_{4}$. Hence no of the $\hat{\tau}_{i}, i=1,2,3,4$ is greater than $\left(\hat{\tau}_{1} \cap \hat{\tau}_{2}\right) \vee\left(\hat{\tau}_{3} \cap \hat{\tau}_{4}\right)$ and it suffices to use Lemma 3.

Condition b): Proving indirectly let us suppose that $T(H)$ contains two incomparable elements $\hat{\tau}^{\prime}, \hat{\tau}^{\prime \prime}$ such that there exist the elements $\hat{\tau}_{n}^{\prime}, \hat{\tau}_{n}^{\prime \prime}$ in $T(H)$ satisfying $\hat{\tau}_{n}^{\prime} \cap \hat{\tau}_{n}^{\prime \prime}=\hat{\tau}_{n}, \hat{\tau}_{n}>\hat{\tau}_{n+1}>\hat{\tau}=\hat{\tau}^{\prime} \cap \hat{\tau}^{\prime \prime}, n=1,2, \ldots$ By Lemma 8 we can clearly assume that all the $\hat{\tau}_{n}^{\prime \prime}$ are equal to the same $\hat{\tau}_{0}^{\prime}$. Let $J_{0}, J_{1}, J_{2}, \ldots$ be the rank one direct summands of $H$ (of a given complete decomposition) of the types $\hat{\tau}_{0}^{\prime}, \hat{\tau}_{1}^{\prime}, \hat{\tau}_{2}^{\prime}, \ldots$ and let $u_{i} \in J_{i}, i=0,1,2, \ldots$ be arbitrary non-zero elements. By hypothesis, the pure subgroup $S=\left\{u_{0}-u_{i}, i=1,2, \ldots\right\}_{*}^{H}$ of $H$ is completely decomposable.

From the linear independence of the elements $u_{0}, u_{1}, u_{2}, \ldots$ it easily follows that $S \cap J_{i}=0, i=0,1,2, \ldots$ so that by Lemma $9 S$ contains the elements of the types $\hat{\tau}_{1}, \hat{\tau}_{2}, \ldots$, only. The types $\hat{\tau}^{\prime}, \hat{\tau}^{\prime \prime}$ cannot be both comparable with infinitely many $\hat{\tau}_{n}$ since they are incomparable $\left(\hat{\tau}^{\prime}<\hat{\tau}_{n}, \hat{\tau}^{\prime \prime}<\hat{\tau}_{n}\right.$ cannot happen by a) while the other possibilities lead to the comparability of $\left.\hat{\tau}^{\prime}, \hat{\tau}^{\prime \prime}\right)$. Therefore one of the $\hat{\tau}^{\prime}, \hat{\tau}^{\prime \prime}$ is incomparable with infinitely many $\hat{\tau}_{n}$. Without loss of generality we can assume $\hat{\tau}^{\prime} \| \hat{\tau}_{n}$, $n=1,2, \ldots$ Let $J^{\prime}$ be a rank one direct summand of $H$ of a given complete decomposition of the type $\hat{\tau}^{\prime}$. Since $\hat{\tau}^{\prime} \neq \hat{\tau}_{n}^{\prime}, n=0,1,2, \ldots$ we have that the subgroup $S+J^{\prime}$ of $H$ is pure in $H$. By applying of Lemma 5 to $S+J^{\prime}$ we get a contradiction.

Condition c): First, let us have two incomparable elements $\hat{\tau}_{1}, \hat{\tau}_{2}$ from $T(H)$. For $\hat{\tau}>\hat{\tau}_{1}$ we have $\hat{\tau}_{1}=\hat{\tau} \cap \hat{\tau}_{1}>\hat{\tau} \cap \hat{\tau}_{1} \cap \hat{\tau}_{2}=\hat{\tau}_{1} \cap \hat{\tau}_{2}=\hat{\tau} \cap \hat{\tau}_{2}$ by Lemma 2 and it suffices to use Lemma 6 . Now let $\hat{\tau}_{1}, \hat{\tau}_{2}, \hat{\tau}_{3}$ be three different elements from $T(H), \hat{\tau}_{1} \cap \hat{\tau}_{2} \| \hat{\tau}_{3}$ and let $\hat{\tau}^{\prime} \in T(H)$ be an element satisfying $\hat{\tau}^{\prime} \geqq \hat{\tau}_{1} \cap \hat{\tau}_{2}$. Using Lemma 7 we have $\hat{\tau}^{\prime} \cap \hat{\tau}_{2} \geqq \hat{\tau}_{1} \cap \hat{\tau}_{2}>\hat{\tau}_{1} \cap \hat{\tau}_{2} \cap \hat{\tau}_{3}=\hat{\tau}_{1} \cap \hat{\tau}_{3}=\hat{\tau}_{2} \cap \hat{\tau}_{3}$ and $\hat{\tau}^{\prime} \cap \hat{\tau}_{2}>\hat{\tau} \cap \hat{\tau}_{3}=\hat{\tau}_{2} \cap \hat{\tau}_{3}=\hat{\tau}_{1} \cap \hat{\tau}_{2} \cap \hat{\tau}_{3}$ from which it easily follows that any type $\hat{\tau} \in \hat{\tau}\left(H\left(\hat{\tau}_{1} \cap \hat{\tau}_{2}\right)\right)$ has with $\hat{\tau}_{3}$ the same intersection $\hat{\tau}_{1} \cap \hat{\tau}_{2} \cap \hat{\tau}_{3}$. Hence $H\left(\hat{\tau}_{1} \cap \hat{\tau}_{2}\right)$ is necessarily of finite rank by Lemma 6 .

Condition $\mathrm{d}$ ): Proving indirectly let us suppose that the set of all maximal elements of $T(H)$ is uncountable.

At first, let us assume that no element of $T(H)$ is comparable with all other elements of $T(H)$. In this case we consider two cases:

a) $\hat{\tau}(H)$ does not satisfy the minimum condition. Let $\hat{\tau}_{1}>\hat{\tau}_{2}>\ldots$ be any infinite decreasing sequence of elements of $\hat{\tau}(H)$ and let $\hat{\tau}$ be any element of $T(H)$. The inequality $\hat{\tau}<\hat{\tau}_{n}, n=1,2, \ldots$ is clearly impossible. If $\hat{\tau}$ is incomparable with in- 
finitely many $\hat{\tau}_{n}$ then using Lemma 10 we get a contradiction by applying of the same method as in b). Therefore $\hat{\tau} \geqq \hat{\tau}_{n}$ for some $n$ and $H=\bigcup_{n=1}^{\infty} H\left(\hat{\tau}_{n}\right)$. Any $H\left(\hat{\tau}_{n}\right)$ is of finite rank by c) so that $H$ is countable contradicting to our hypothesis.

B) $\hat{\tau}(H)$ satisfies the minimum condition. In this case $\hat{\tau}(H)$ contains the least element $\hat{\tau}$ since the intersection of two minimal elements of $\hat{\tau}(H)$ lies in $\hat{\tau}(H)$. Let $\left\{\hat{\tau}_{\alpha}, \alpha \in A\right\}$ be the set of all minimal elements of the set $\left\{\hat{\tau}^{\prime}, \hat{\tau}^{\prime} \in \hat{\tau}(H), \hat{\tau}^{\prime}>\hat{\tau}\right\}$. We shall divide the index set $A$ into two disjoint subsets $A_{1}, A_{2}$ in such a way that $\alpha \in A_{1}$ iff $\hat{\tau}_{\alpha} \in T(H)$ and $\alpha \in A_{2}$ iff $\hat{\tau}_{\alpha} \notin T(H)$. If $\alpha \neq \beta$ are elements from $A_{2}$, then $\hat{\tau}_{\alpha}=$ $=\hat{\tau}_{\alpha}^{\prime} \cap \hat{\tau}_{\alpha}^{\prime \prime}, \hat{\tau}_{\beta}=\hat{\tau}_{\beta}^{\prime} \cap \hat{\tau}_{\beta}^{\prime \prime}, \hat{\tau}_{\alpha}^{\prime}, \hat{\tau}_{\alpha}^{\prime \prime}, \hat{\tau}_{\beta}^{\prime}, \hat{\tau}_{\beta}^{\prime \prime} \in T(H)$ by Lemma 10, and the condition a) implies $\hat{\tau}_{\alpha}^{\prime}\left\|\hat{\tau}_{\beta}^{\prime}, \hat{\tau}_{\alpha}^{\prime}\right\| \hat{\tau}_{\beta}^{\prime \prime}, \hat{\tau}_{\alpha}^{\prime \prime}\left\|\hat{\tau}_{\beta}^{\prime}, \hat{\tau}_{\alpha}^{\prime \prime}\right\| \hat{\tau}_{\beta}^{\prime \prime}$. By the condition c) just proved, every $H\left(\hat{\tau}_{\alpha}\right)$, $\alpha \in A$ is of finite rank so that $A$ is uncountable by the condition a). Now we can take rank one direct summands $J_{\alpha}, \alpha \in A_{1}, J_{\beta}^{\prime}, J_{\beta}^{\prime \prime}, \beta \in A_{2}$ of a given complete decomposition of $H$ such that $\hat{\tau}\left(J_{\alpha}\right)=\hat{\tau}_{\alpha}, \hat{\tau}\left(J_{\beta}^{\prime}\right)=\hat{\tau}_{\beta}^{\prime}, \hat{\tau}\left(J_{\beta}^{\prime \prime}\right)=\hat{\tau}_{\beta}^{\prime \prime}$. If $u_{\beta}^{\prime} \in J_{\beta}^{\prime}, u_{\beta}^{\prime \prime} \in J_{\beta}^{\prime \prime}, \beta \in A_{2}$ are arbitrary non-zero elements then $S=\sum_{\alpha \in A_{1}} J_{\alpha}+\sum_{\beta \in A_{2}}\left\{u_{\beta}^{\prime}+u_{\beta}^{\prime \prime}\right\}_{*}^{H}$ is a pure subgroup of $H$ and Lemma 12 yields a contradiction.

In the general case let there exist elements in $T(H)$ comparable with any other elements of $T(H)$ and let $\hat{\tau}$ be a maximal one with this property. If we put $H_{1}=\sum_{\alpha \in A_{1}} J_{\alpha}$ where $\hat{\tau}\left(J_{\alpha}\right)>\hat{\tau}$ and $H_{2}=\sum_{\alpha \in A_{2}} J_{\alpha}$ where $\hat{\tau}\left(J_{\alpha}\right) \leqq \hat{\tau}$ then the set of all maximal elements of $T(H)$ coincides with the set of all maximal elements of $T\left(H_{1}\right)$ and hence it is countable by the preceding part.

The proof of the necessity is therefore finished.

2) Sufficiency: We divide this part into several cases:

a) $G$ is a reduced group of finite rank and no element from $T(G)$ is comparable with all other elements from $T(G)$. In this case $\hat{\tau}(G)$ contains the least element $\hat{\tau}$. Let $\hat{\tau}_{1}(G)$ be the set of all maximal elements of $\hat{\tau}(G)$. If $\hat{\tau}_{1}(G), \hat{\tau}_{2}(G), \ldots, \hat{\tau}_{k}(G)$ are defined then let $\hat{\tau}_{k+1}(G)$ be the set of all maximal elements of $\hat{\tau}(G)$ not belonging to $\hat{\tau}_{1}(G) \cap \hat{\tau}_{2}(G) \cup \ldots \cup \hat{\tau}_{k}(G)$. In view of our hypothesis there exists a natural integer $n$ such that $\hat{\tau}_{n}(G)=\hat{\tau}$. Now we shall use the induction by $n$. For $n=1 G$ is homogeneous of the type $\hat{\tau}$ and therefore any its pure subgroup is completely decomposable by Theorem 46.6 in [1]. For $n>1$ let $\hat{\tau}_{1}, \hat{\tau}_{2}, \ldots, \hat{\tau}_{k}$ be all the minimal elements of the set $\left\{\hat{\tau}^{\prime}, \hat{\tau}^{\prime} \in \hat{\tau}(G), \hat{\tau}^{\prime}>\hat{\tau}\right\}$ and let $S$ be any pure subgroup of $G$. Any $G\left(\hat{\tau}_{i}\right), i=1,2, \ldots$ $\ldots, k$ is a direct sum of those rank one direct summands $J_{\alpha}$ of a given complete decomposition of $G$ the types of which are greater than or equal to $\hat{\tau}_{i}$. By hypothesis, $\hat{\tau} \notin T(G)$ so that by a) and Lemma 12 we have $G=\sum_{i=1}^{k} G\left(\hat{\tau}_{i}\right)$. Let $\hat{\tau}_{i}^{\prime}>\hat{\tau}_{i}, \hat{\tau}_{j}^{\prime}>\hat{\tau}_{j}, i \neq j$ be two elements from $\hat{\tau}(G)$. By a) and Lemma 7 we have $\hat{\tau}_{i}^{\prime} \cap \hat{\tau}_{i}>\hat{\tau}_{\boldsymbol{i}} \cap \hat{\tau}_{\boldsymbol{j}}=\hat{\tau}_{\boldsymbol{i}}^{\prime} \cap \hat{\tau}_{\boldsymbol{j}}$ and 
$\hat{\tau}_{j}^{\prime} \cap \hat{\tau}_{j}>\hat{\tau}_{\boldsymbol{i}} \cap \hat{\tau}_{j}=\hat{\tau}_{\boldsymbol{i}}^{\prime} \cap \hat{\tau}_{\boldsymbol{j}}=\hat{\tau}_{\boldsymbol{i}}^{\prime} \cap \hat{\tau}_{j}^{\prime}$. Therefore Lemma 17 yields $S \cap\left(G\left(\hat{\tau}_{1}\right)+\ldots\right.$ $\left.\ldots+G\left(\hat{\tau}_{i+1}\right)\right)=S \cap\left(G\left(\hat{\tau}_{1}\right)+\ldots+G\left(\hat{\tau}_{i}\right)+S \cap G\left(\tau_{i+1}\right)+T_{i}, i=1,2, \ldots, k-1\right.$, where $T_{i}$ is completely decomposable. From this it immediately follows that $S=$ $=S \cap G=\sum_{i=1}^{\infty} S \cap G\left(\hat{\tau}_{i}\right)+\sum_{i=1}^{k-1} T_{i}$. Hence $S$ is completely decomposable since $S \cap G\left(\hat{\tau}_{i}\right), i=1,2, \ldots, k$ are so by the induction hypothesis.

$\beta) G$ is a reduced group, no element from $T(G)$ is comparable with all other elements from $T(G)$ and $\hat{\tau}(G)$ satisfies the minimum condition. In this case $\hat{\tau}(G)$ clearly contains the least element $\hat{\tau}$. The set $M$ of all minimal elements of the set of all types from $\hat{\tau}(G)$ greater than $\hat{\tau}$ is at most countable by the conditions a) and $\mathrm{d})$. Assuming $M$ finite we are led to the case $\alpha$ ) owing to the condition a), and Lemma 10. Hence we can assume that $M=\left\{\hat{\tau}_{1}, \hat{\tau}_{2}, \ldots\right\}$ is infinite countable. If $S$ is a pure subgroup of $G$ then as in the part $\alpha)$ we have $G=\sum_{i=1}^{\infty} G\left(\hat{\tau}_{i}\right)$ and $S \cap\left(G\left(\hat{\tau}_{1}\right)+\ldots+G\left(\hat{\tau}_{i+1}\right)\right)=$ $=S \cap\left(G\left(\hat{\tau}_{1}\right)+\ldots+G\left(\hat{\tau}_{i}\right)+S \cap G\left(\tau_{i+1}\right)+T_{i}, i=1,2, \ldots\right.$ The usual routine in the theory of completely decomposable groups gives $S=\sum_{i=1}^{\infty} S \cap G\left(\hat{\tau}_{i}\right)+\sum_{i=1}^{\infty} T_{i}$ which finishes the proof of this part since any $S \cap G\left(\hat{\imath}_{i}\right), i=1,2, \ldots$ is completely decomposable by the part $\alpha$ ).

$\gamma) G$ is a reduced group, no element from $T(G)$ is comparable with all other elements from $T(G)$ and $\hat{\tau}(G)$ does not satisfy the minimum condition. Let $\hat{\tau}_{1}>\hat{\tau}_{2}>\ldots$ be an infinite decreasing sequence of elements of $\hat{\tau}(G)$. If $\hat{\tau}_{n+1} \in T(G)$ then there is $\hat{\tau} \in T(G), \hat{\tau} \| \hat{\tau}_{n+1}$. $\hat{\tau}>\hat{\tau}_{n}$ is clearly impossible and $\hat{\tau}>\hat{\tau}_{n}$ is impossible by the condition a) (and Lemma 10). Thus $\hat{\tau} \| \hat{\tau}_{n}$. If $\hat{\tau}_{n+1} \notin T(G)$, then $\hat{\tau}_{n+1}=\hat{\tau}_{n+1}^{\prime} \cap \hat{\tau}_{n+1}^{\prime \prime}$, where $\hat{\tau}_{n+1}^{\prime}, \hat{\tau}_{n+1}^{\prime \prime} \in T(G)$ by Lemma 10 and $\hat{\tau}_{n+1}^{\prime} \| \hat{\tau}_{n+1}^{\prime \prime}$. It is easy to see that at least one of the elements $\hat{\tau}_{n+1}^{\prime}, \hat{\tau}_{n+1}^{\prime \prime}$ is incomparable with $\hat{\tau}_{n}$. Hence $G\left(\hat{\tau}_{n}\right)$ is of finite rank by the condition c). Since $G\left(\hat{\tau}_{n}\right)$ has a finite type set we can assume $\hat{\tau}_{n+1}$ is the maximal element in the set of all elements from $\hat{\tau}(G)$ less than $\hat{\tau}_{n}$ (it is unique by Lemma 11). If $\hat{\tau}_{n, 1}, \hat{\tau}_{n, 2}, \ldots, \hat{\tau}_{n, k}$ are all the minimal elements of the set of all elements of $\hat{\tau}(G)$ greater than or equal to $\hat{\tau}_{n}$ then one of them, say $\hat{\tau}_{n, 1}$, is $\hat{\tau}_{n-1}$. The part $\alpha$ ) shows that $S \cap G\left(\hat{\tau}_{n}\right)$ is a direct sum of $S \cap G\left(\hat{\tau}_{n-1}\right)$ and a completely decomposable group $T_{n-1}$. Further, no element $\hat{\tau}$ from $T(G)$ can be less than all the $\hat{\tau}_{n}$ owing to the condition $b$ ). If $\hat{\tau}$ is incomparable with infinitely many $\hat{\tau}_{n}$ then we can without loss of generality assume that $\hat{\tau} \| \hat{\tau}_{n}, n=1,2, \ldots$ Lemma 7 yields $\hat{\tau}_{n}=\hat{\tau}_{1} \cap \hat{\tau}_{n}>\hat{\tau}_{n} \cap \hat{\tau}=\hat{\tau}_{1} \cap \hat{\tau}$ which contradicts to the condition $\mathrm{b}$ ). Therefore $\hat{\tau}>\hat{\tau}_{n}$ for some $n$ and $G=\bigcup_{n=1}^{\infty} G\left(\hat{\tau}_{n}\right)$. As in the preceding part the usual routine yields $S=S \cap G\left(\hat{\tau}_{1}\right)+\sum_{i=1}^{\infty} T_{i}$ which finishes the proof of this part.

$\delta) G$ is reduced. Let $\hat{\tau}$ be the maximal element of the set of all elements of $T(G)$ which are comparable with any other element of $T(G)$. If $G_{1}$ is the direct sum of 
those rank one direct summands of a given complete decomposition of $G$ the type of which is greater than $\hat{\tau}$ and $G_{2}$ is the complementary direct summand then $\hat{\tau}\left(G_{2}\right)$ is clearly inversely well-ordered by a) and $\hat{\tau}_{1} \geqq \hat{\tau}_{2}$ for $\hat{\tau}_{i} \in \hat{\tau}\left(G_{i}\right), i=1,2$. If $S$ is a pure subgroup of $G$ then $S$ is a direct sum of $S \cap G_{1}$ and a completely decomposable group by Wang's theorem. $S \cap G_{1}$ is pure in $G_{1}$ so that it suffices to use the part $\beta$ ) resp. $\gamma$ ).

ع) $G=D+H$ where $D$ is divisible and $H$ reduced. If $S$ is a pure subgroup of $G$ then we can assume that $S=S \cap D+S^{\prime}, S^{\prime} \subseteq H$ owing the well-known properties of divisible groups (see $[1] \S 18$, p. 63 ). Here $S^{\prime}$ is clearly pure in $H$ so that it suffices to use the part $\delta$ ).

The proof of Theorem 1 is therefore complete.

Now we are going to the general case.

Lemma 18. Let $G$ be a reduced completely decomposable group of the form $G=J+\sum_{i=1}^{\infty} J_{i}$ where $J, J_{i}, i=1,2, \ldots$ are of rank one and $\hat{\tau}_{1}=\hat{\tau}\left(J_{1}\right)<\hat{\tau}_{2}=$ $=\hat{\tau}\left(J_{2}\right)<\ldots<\hat{\tau}_{n}=\hat{\tau}\left(J_{n}\right)<\ldots<\hat{\tau}=\hat{\tau}(J)$. Then $G$ contains a pure subgroup which is not completely decomposable.

Proof. Let $p$ be a prime for which $J$ (and hence $J_{1}, J_{2}, \ldots$ ) is not $p$-divisible. Let us take the elements $0 \neq u \in J, 0 \neq u_{i} \in J_{i}, i=1,2, \ldots$, with $h_{p}^{G}(u)=h_{p}^{G}\left(u_{i}\right)=0$, $i=1,2, \ldots$ and let us put $S=\left\{u+p u_{1}, u+p u_{2}, \ldots, u+p u_{n}, \ldots\right\}_{*}^{G}$. If $S$ is completely decomposable then $S$ can be written in the form $S=\sum_{n=1}^{\infty} S_{n}$ where $S_{n}$ is a homogeneous completely decomposable group of the type $\hat{\tau}_{n}^{\prime}$, and $\hat{\tau}_{n}^{\prime} \leqq \hat{\tau}_{n+1}^{\prime}, n=1,2, \ldots$ Then $u+p u_{1} \in H_{1}=S_{1}+S_{2}+\ldots+S_{k}$. By hypothesis there exists an element $u+p u_{i}$ the type of which is greater than $\hat{\tau}_{k}$ so that $u+p u_{i} \in H_{2}=\sum_{j=k+1}^{\infty} S_{j}$. Further, $p\left(u_{1}-u_{i}\right)=\left(u+p u_{1}\right)-\left(u+p u_{i}\right) \in S$. Hence $u_{1}-u_{i} \in S, S$ being pure in $G$. Therefore $u_{1}-u_{i}=h_{1}+h_{2}, h_{1} \in H_{1}, h_{2} \in H_{2}$ from which $p\left(u_{1}-u_{i}\right)=$ $p h_{1}+p h_{2}=\left(u+p u_{1}\right)-\left(u+p u_{i}\right)$. From the form of $S$ it follows $p h_{1}=u+p u_{1}$ which yields a contradiction with $h_{p}^{G}(u)=0$.

Let us denote by $\mathfrak{M}$ the class of all completely decomposable groups $G$ having two following properties:

1) the type set of $G$ contains an infinite increasing sequence $\hat{\tau}_{1}<\hat{\tau}_{2}<\ldots$ such that for every $\hat{\tau} \in \hat{\tau}(G)$ there is $\hat{\tau} \leqq \hat{\tau}_{n}$ for some $n$ and the set $\left\{\hat{\tau}, \hat{\tau} \in \hat{\tau}(G), \hat{\tau} \leqq \hat{\tau}_{n}\right\}$ is inversely well-ordered for every $n$,

2) any pure subgroup of $G$ is completely decomposable.

Theorem 2. Any pure subgroup of a completely decomposable torsion free group G is completely decomposable if and only if either $\hat{\tau}(G)$ satisfies the maximum con- 
dition and therefore $G$ is of the form of Theorem 1 , or $G$ is of the form $G=D+$ $\dot{+} G_{1}+G_{2}$ where $D$ is divisible, $G_{1} \in \mathfrak{M}, T\left(G_{2}\right)$ is inversely well-ordered and $\hat{\tau}_{1} \geqq \hat{\tau}_{2}$ for any $\hat{\tau}_{i} \in \hat{\tau}\left(G_{i}\right), i=1,2$.

Proof. For to prove the necessity we can clearly restrict ourselves to the case $G$ reduced and the type set $\hat{\tau}(G)$ of $G$ does not satisfy the maximum condition. Thus let $\hat{\tau}_{1}<\hat{\tau}_{2}<\ldots$ be an increasing sequence of elements of $\hat{\tau}(G)$ and let $\hat{\tau} \in T(G)$, $\hat{\tau}>\hat{\tau}_{n}$ for all $n=1,2, \ldots$ If infinitely many of $\hat{\tau}_{n}$ ly in $T(G)$ then Lemma 18 gives a contradiction. Thus we can assume no $\hat{\tau}_{n}$ lies in $T(G)$. Since the condition a) from Theorem 1 does not depend on the maximum condition of the type set $\hat{\tau}(G)$ we can use Lemma 10. Thus for every $n=1,2, \ldots$ there are elements $\hat{\tau}_{n}^{\prime}, \hat{\tau}_{n}^{\prime \prime}$ in $T(G)$ such that $\hat{\tau}_{n}^{\prime} \cap \hat{\tau}_{n}^{\prime \prime}=\hat{\tau}_{n}, \hat{\tau}_{n}^{\prime} \| \hat{\tau}_{n}^{\prime \prime}$. By Lemma 7 we have either $\hat{\tau} \cap \hat{\tau}_{n}^{\prime} \cap \hat{\tau}_{n}^{\prime \prime}=\hat{\tau} \cap \hat{\tau}_{n}^{\prime}=\hat{\tau}_{n}^{\prime} \cap \hat{\tau}_{n}^{\prime \prime} \leqq$ $\leqq \hat{\tau} \cap \hat{\tau}_{n}^{\prime \prime}$ or $\hat{\tau} \cap \hat{\tau}_{n}^{\prime} \cap \hat{\tau}_{n}^{\prime \prime}=\hat{\tau} \cap \hat{\tau}_{n}^{\prime \prime}=\hat{\tau}_{n}^{\prime} \cap \hat{\tau}_{n}^{\prime \prime} \leqq \hat{\tau} \cap \hat{\tau}_{n}^{\prime}$ so that we can assume $\hat{\tau} \cap \hat{\tau}_{n}^{\prime}=$ $=\hat{\tau}_{n}^{\prime} \cap \hat{\tau}_{n}^{\prime \prime}=\hat{\tau}_{n}$. As is easy to see, $G$ satisfies the condition c) of Theorem 1 , so that $G\left(\hat{\tau}_{n}^{\prime}\right)$ is of finite rank and we can assume $\hat{\tau}_{k}^{\prime} \leqq \hat{\tau}_{n}^{\prime}$ for no $k<n$. Let us suppose $\hat{\tau}_{n}^{\prime}<\hat{\tau}_{k}^{\prime}$ for some $k<n$. Then $\hat{\tau}_{k}=\hat{\tau} \cap \hat{\tau}_{k}^{\prime}=\hat{\tau}_{k}^{\prime} \cap \hat{\tau}_{k}^{\prime \prime}$ and $\hat{\tau}_{n}=\hat{\tau} \cap \hat{\tau}_{n}^{\prime}=\hat{\tau}_{n}^{\prime} \cap \hat{\tau}_{n}^{\prime \prime}$ and $\hat{\tau}_{k}=$ $=\hat{\tau}_{k} \cap \hat{\tau}_{n}=\hat{\tau} \cap \hat{\tau}_{k}^{\prime} \cap \hat{\tau}_{n}^{\prime}=\hat{\tau} \cap \hat{\tau}_{n}=\hat{\tau}_{n}$, a contradiction showing $\hat{\tau}_{n}^{\prime}$ are pairwise incomparable. Now for $n>k$ Lemma 7 gives $\hat{\tau}_{n}=\hat{\tau} \cap \hat{\tau}_{n}^{\prime}>\hat{\tau}_{k}=\hat{\tau} \cap \hat{\tau}_{k}^{\prime}=\hat{\tau}_{k}^{\prime} \cap \hat{\tau}_{n}^{\prime}$. We choose the rank one direct summands $J, J_{n}$ of $G$ such that $\hat{\tau}\left(J_{n}\right)=\hat{\tau}_{n}^{\prime}, \hat{\tau}(J)=\hat{\tau}$, $n=1,2, \ldots$ For $0 \neq u_{n} \in J_{n}$ we consider the pure subgroup $S^{\prime}=\left\{u_{1}-u_{2}\right.$, $\left.u_{2}-u_{3}, \ldots\right\}_{*}^{G}$. By the above the type set of $S^{\prime}$ is just $\left\{\hat{\tau}_{1}, \hat{\tau}_{2}, \ldots\right\}$. Since $S^{\prime}$ is completely decomposable we can select a direct summand $S$ of $S^{\prime}+J$ satisfying all the conditions of Lemma 18. The obtained contradiction shows that no element of $T(G)$ can be greater than some infinite increasing sequence of elements of $\hat{\tau}(G)$.

Now let $\hat{\tau}(G)$ contains an infinite increasing sequence $\hat{\tau}_{1}<\hat{\tau}_{2}<\ldots$ and let $\hat{\tau} \in \hat{\tau}(G)$ be an arbitrary element. If $\hat{\tau} \in T(G)$ then $\hat{\tau} \| \hat{\tau}_{1}$ is impossible by the condition c) of Theorem 1. If $\hat{\tau} \notin T(G)$ then $\hat{\tau}=\hat{\tau}^{\prime} \cap \hat{\tau}^{\prime \prime}, \hat{\tau}^{\prime} \| \hat{\tau}^{\prime \prime}, \hat{\tau}^{\prime}, \hat{\tau}^{\prime \prime} \in T(G)$ by Lemma 10. No of $\hat{\tau}^{\prime}, \hat{\tau}^{\prime \prime}$ is greater than all $\hat{\tau}_{n}$ by the preceding part and $\hat{\tau}^{\prime}<\hat{\tau}_{n}, \hat{\tau}^{\prime \prime}<\hat{\tau}_{n}$ is impossible by the condition a) of Theorem 1 . Thus at least one of the $\hat{\tau}^{\prime}, \hat{\tau}^{\prime \prime}$ is incomparable with infinitely many $\hat{\tau}_{n}$, which contradicts to the condition c) of Theorem 1 . Hence either $\hat{\tau}<\hat{\tau}_{1}$ or $\hat{\tau} \geqq \hat{\tau}_{1}$. We can write $G=G_{1}+G_{2}$ where $G_{1}=G\left(\hat{\tau}_{1}\right)$ and $G_{2}$ is the complementary direct summand. By the preceding part, for every $\hat{\tau}_{i} \in \hat{\tau}\left(G_{i}\right), i=1,2$ there is $\hat{\tau}_{1}>\hat{\tau}_{2} . \hat{\tau}\left(G_{2}\right)$ is ordered by the condition a) of Theorem 1 and it is inversely well-ordered by the beginning of this proof. By the above, every $\hat{\tau}>\hat{\tau}_{1}$ is comparable with any $\hat{\tau}_{n}$ so that $\hat{\tau}\left(G_{1}\right)$ is ordered and the necessity follows easily.

The sufficiency follows from Theorem 1 in the first case while in the second case any pure subgroup of $G$ can be written in the form $S=S \cap D+S^{\prime}, S^{\prime} \subseteq G_{1}+G_{2}$ (by the well-known properties of divisible groups). Since $S^{\prime}$ is pure in $G_{1}+G_{2}$, Wang's theorem implies that $S^{\prime}$ is a direct sum of $S^{\prime} \cap G_{1}$ and a completely decomposable group. Finally $S^{\prime} \cap G_{1}$ is pure in $G_{1}$, hence $S^{\prime} \cap G_{1}$ is completely decomposable by hypothesis and we are ready. 


\section{References}

[1] L. Fuchs: Abelian groups, Budapest 1966.

[2] John S. P. Wang: On completely decomposable groups, Proc. Amer. Math. Soc. 15 (1964), $184-186$.

[3] L. Procházka: Generalization of a theorem of R. Baer, Comment. Math. Univ. Carol. 4 (1963), 105-108.

[4] L. Bican: On isomorphism of quasi-isomorphic torsion free Abelian groups, Comment. Math. Univ. Carol. 9 (1968), 109-119.

[5] L. Fuchs: Notes on abelian groups I, Ann. Univ. Sci. Bud. 2 (1959), 5-23.

[6] L. Procházka: О расщепляемости фактор-групп абелевых групп без кручения конечного paнra, Czech. Math. J. 11 (86), (1961), 521-557.

[7] E. S. Ljapin: О разложении абелевых групп в прямые суммы рациональных групп, Мат. сб. 8 (1940), 205-237.

Author's address: 18600 Praha 8-Karlín, Sokolovská 83, ČSSR (Matematicko-fyzikální fakulta UK). 